\title{
Experimental evidences of the effectiveness of some liquefaction mitigation measures
}

\author{
Emilio Bilotta, Anna Chiaradonna, Gianluca Fasano, Alessandro Flora, Lucia Mele, Valeria Nappa \\ University of Napoli Federico II, Italy
}

Stefania Lirer

University Guglielmo Marconi, Roma, Italy

Vincenzo Fioravante

University of Ferrara, Italy - ISMGEO S.p.a

Contacting author: bilotta@unina.it

\begin{abstract}
Earthquakes induced liquefaction is one of the most significant causes of damage to structures during an earthquake. Ground improvement is currently considered to be the most appropriate mitigation technique to prevent soil liquefaction. This work is aimed to compare different liquefaction mitigation techniques for protection of small-to-medium sized 'critical' infrastructures and low-rise buildings. The effectiveness of some techniques (densification, addition of fine contents, induced partial saturation and drainage systems) was verified via experimental evidences coming from laboratory testing, physical modelling and liquefaction field prototype tests. Starting from the calibration of advanced soil constitutive models, numerical modelling activities were extended to the back-analysis of centrifuge tests and field prototype tests, up to a parametric study with different geometrical layouts.
\end{abstract}

Keywords: soil liquefaction; mitigation techniques, laboratory tests, numerical modelling, centrifuge tests.

\section{Introduction}

Excessive deformation of ground surface caused by earthquakes are of great concern for civil engineering works, human lives and the environmental. Such ground deformations are often associated with soil liquefaction. Liquefaction is a phenomenon marked by a rapid loss of soil strength which can occur in loose, saturated soil deposits subjected to earthquake shaking or other forms of rapid loading. During liquefaction, when the effective stresses approach zero, soil behaviour switches from that of a solid to that of a fluid, causing serious damage to engineering structures.

Ground improvement is currently considered to be the most appropriate approach to mitigate soil liquefaction. As part of the objective of the LIQUEFACT (WP4) project is to make an objective comparison of the different mitigation techniques. Not all the existing techniques can be applied in densely urbanized areas or sites with historic buildings to be preserved. In these cases, it becomes necessary to adopt less invasive mitigation techniques that combine the need to reduce the risk of liquefaction and the protection of the integrity of the existing buildings or critical infrastructures.

In this framework, the effectiveness of some techniques have been experimentally analysed:

- Soil densification;

- Addition of fine content;

- induced partial saturation; 
- Drainage system.

The most effective ones, both from the point of view of laboratory performance and costs, have been tested in physical models in a seismic centrifuge (ISMGEO) and in atrial field located in Emilia Romagna region (TREVI s.p.A).

\section{Experimental activities}

A large experimental programme has been planned in order to carry out an exhaustive study of the mechanical behaviour under cyclic stress paths of liquefiable soils, treated with different mitigation techniques. To this aim, cyclic triaxial tests and cyclic simple shear tests have been performed on reconstituted (Leighton Buzzard sand-LB, Pieve di Cento sand-PdC, Sant'Agostino sand-SAS, Bauxite, Inagisand, Fig.1) and undisturbed (Pieve di Cento sand) specimens of sandy soils, prior and after treatments (densification, addition of fine, low desatutarion). The PdC and SAS soils have been recovered in Emilia Romagna region (Italy) which was affected by liquefaction in 2012 earthquake. In the same time, centrifuge tests have been carried out at the ISMGEO laboratory (Istituto Sperimentale Modelli Geotecnici, Italy) with the goal to analyse the effectiveness of some mitigation techniques: vertical drains (VD), horizontal drains (HD) and Induced Partial Saturation (IPS).

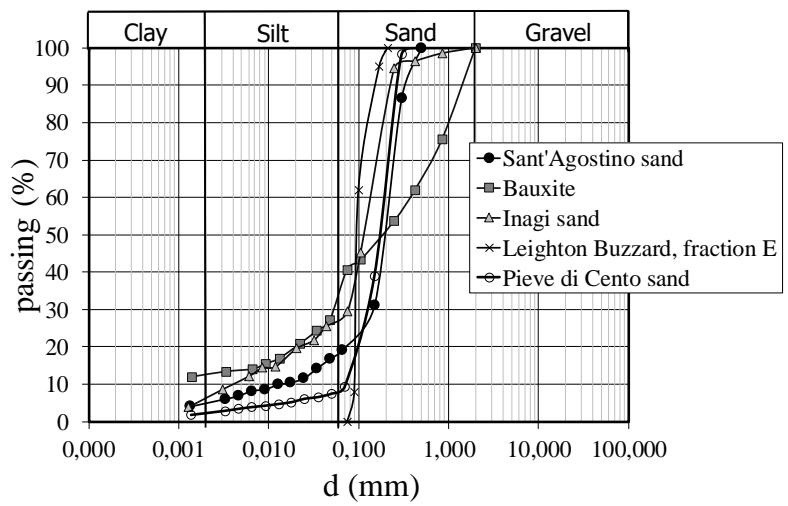

Figure 1. Grain size distribution of tested sands

\subsection{Soil mechanical characterization prior and after treatments}

\subsubsection{Addition of fine content}

Many experimental studies (Ishihara and Koseki 1989; Ishihara 1993, ElMohtar et al. 2014) have shown that the use of mixtures of controlled quantities of highly plastic particles (i.e. bentonite or laponite) can increase the soil liquefaction resistance. The injectability of a mixture within the pores is related to the size of the suspended particles, the initial viscosity of the mixture and its gelling time, tgel (time when its viscosity increases significantly).

The experimental research was carried out on a monogranular sand (Leighton Buzzard LB, $\mathrm{Gs}=2.65)$, manually mixing with dry laponite. In the experimental program, laponite $\mathrm{RD}(\mathrm{Na}+0.7$ [(Si8Mg5.5Li0.3) O20 (OH) 4] -0.7) was adopted. Laponite is a colloidal clay consisting essentially of a mixture of sodium and magnesium silicates, with a structure similar to the montmorillonite one. The particle of laponite has a disk shape with a diameter of $25 \mathrm{~nm}$, thickness of $1 \mathrm{~nm}$ and specific gravity $\mathrm{Gs}=2.57$. The plasticity index $\mathrm{PI}$ is very high: $1100 \%$, and for this reason it is called "superplastic nanoparticle". Laponite particles have numerous negative charges on their surface, which are nominally related to sodium (or magnesium) as a counterion.

The tests were carried out on water/laponite mixtures prepared with two different concentrations ( $\phi=1.5-3.0 \%)$. Due to their low initial viscosity (comparable to that of water), such mixtures can permeate within the pores of the soil without altering its structure.

The results of the undrained cyclic triaxial tests (Fig.2) have confirmed that small amount of laponite ( $1 \%$ of dry weight of sand) can significantly increase the soil liquefaction strength (Mele et al. 2018). The effectiveness of the treatment can be due to two mechanisms: the creation of "bridges" between sand grains due to the plastic nature of laponite, and the formation within the pores of a gel-like fluid that behaves as a material with a small distortion-stiffness (Huang et al. 2016; OchoaCornejo et al. 2016). Both mechanisms reduce the mobility of sand grains during loading cycles, improving the liquefaction resistance. 


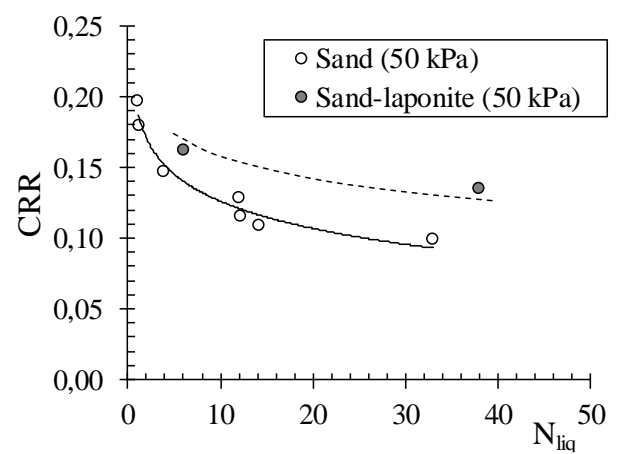

Figure 2. Cyclic resistance curves of $L B$ sand $\left(N_{\text {liq }}\right.$ computed when $R u \geq 0.90)$.

\subsubsection{Induced Partial Saturation IPS}

The Induced Partial Saturation (IPS) increases the resistance to liquefaction of the liquefactionsusceptible soil by introducing some amount of air/gas in the voids. Laboratory results have shown that even a small reduction in the degree of saturation of an initially saturated sand can have a relevant effect (Ishihara et al., 2002; Yegian et al., 2007) on soil liquefaction strength.

The presence of air in the voids increases the resistance against liquefaction in two ways (Okamura and Soga, 2006): the first mechanism is connected to the very low volumetric stiffness of gases, because of which during undrained loading there is a volumetric reduction of the gas phase and therefore reduced excess pore pressures. This mechanism is the ruling one for high degrees of saturation (i.e. dispersed air bubbles). The second mechanism is due to the matric suction of unsaturated soils, which increases the stiffness and strength of soils. This latter mechanism becomes relevant when the degree of saturation is low enough to have a continuous air phase.

The effectiveness of IPS was experimentally analysed via cyclic triaxial tests on saturated (SAS) and unsaturated reconstituted specimens of sandy soils (SAS).

The experimental results (Fig. 3) confirmed that the desaturation increases the liquefaction soil resistance.

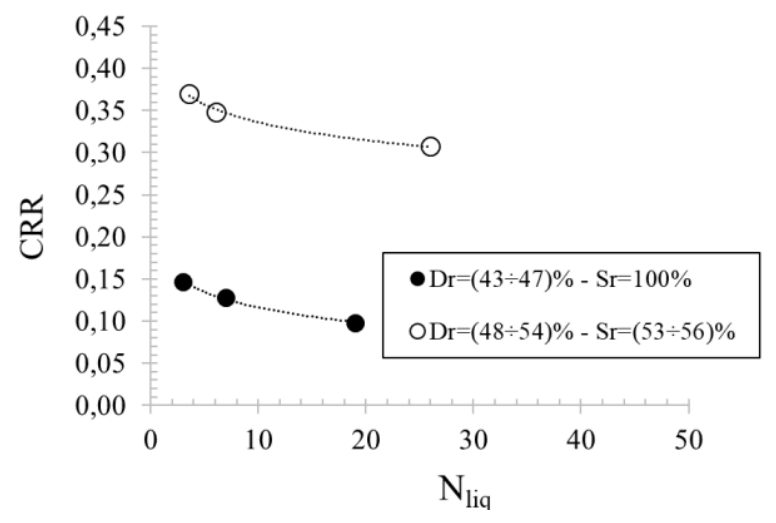

Figure 3. Cyclic resistance curves of SAS ( $N_{\text {liq }}$ computed when $\varepsilon_{D A} \geq 5 \%$ ).

\subsection{Small scale centrifuge tests}

Centrifuge tests were performed at the ISMGEO laboratory. The experimental programme (Tab. 1) was defined with the goal to analyse the behaviour of loose, saturated, sandy deposits, homogeneous or stratified, subjected to increasing seismic excitations up to liquefaction and to verify the effectiveness of three liquefaction mitigation techniques (Fioravante et al., 2019): vertical drains (VD), horizontal drains (HD) and Induced Partial Saturation (IPS). Centrifuge tests were (Fig. 4) carried out in free field conditions or in the presence of a simplified structure placed on the ground surface.

Table 1. Centrifuge tests (ISMGEO).

\begin{tabular}{|c|c|c|c|}
\hline $\begin{array}{l}\text { Boundary } \\
\text { condition }\end{array}$ & Test & one layers & two layers \\
\hline \multirow{3}{*}{ 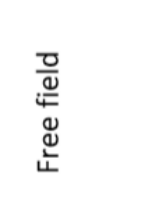 } & reference & $\checkmark$ & $\checkmark$ \\
\hline & $H D, V D$ & $\checkmark$ & $\checkmark$ \\
\hline & IPS & $\checkmark$ & $\checkmark$ \\
\hline \multirow{3}{*}{ 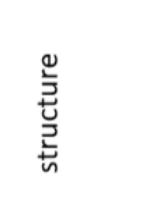 } & reference & $\checkmark$ & $\checkmark$ \\
\hline & $H D, V D$ & $\checkmark$ & $\checkmark$ \\
\hline & IPS & $\checkmark$ & $\checkmark$ \\
\hline
\end{tabular}

\section{Numerical modelling}

Starting from the calibration of advanced soil constitutive models, numerical modelling activities were extended to the back-analysis of centrifuge 
tests and field prototype tests, up to a parametric study with different geometrical layouts.

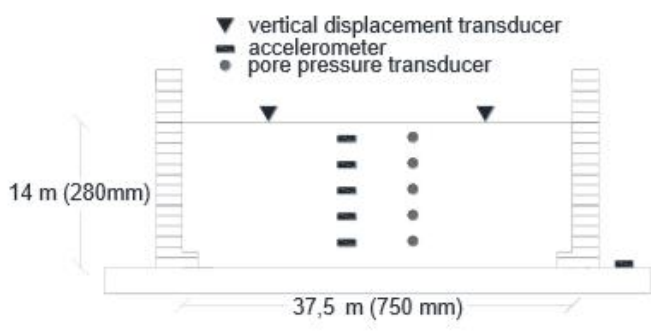

(a)

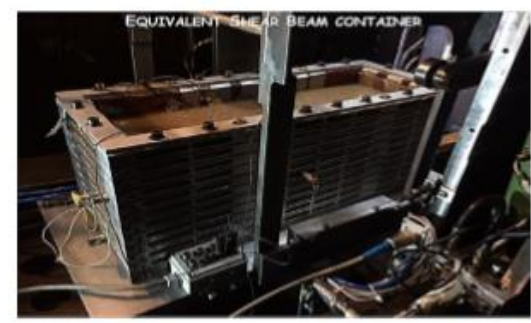

(b)

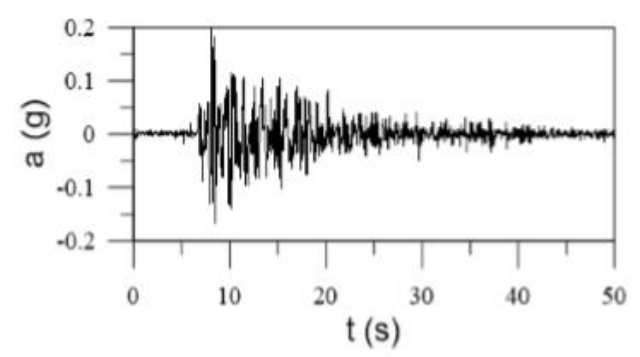

(c)

Figure 4. Layout of the centrifuge model $(a, b)$ and adopted input signal (c).

\subsection{Advanced constitutive model calibration on soil laboratory tests}

Laboratory geotechnical tests have been used to calibrate two advanced constitutive models used in Plaxis2D software: PM4Sand and UBCSand models.

\section{PM4SAND}

The PM4Sand model follows the basic framework of the stress-ratio controlled, critical state compatible, bounding surface plasticity model for sand initially presented by Manzari and Dafalias (1997) and later extended by the same authors. Modifications to the Dafalias- Manzari model were developed and implemented to improve its ability to approximate engineering design relationships that are used to estimate the stressstrain behaviours that are important to predicting liquefaction induced ground deformations during earthquakes. The model is based on effective stresses, respects the critical state theory and uses the relative state parameter indexas defined by Boulanger (2003). This model was implemented in PLAXIS2D in simplified way by casting the various equations and relationships in terms of the inplane stresses only and remove of the Lode angle is in this constitutive model. Bounding, dilatancy and critical surfaces are incorporated in PM4sand following the form of Dafalias and Manzari (2004). A large portion of the post-liquefaction reconsolidation strains are due to the sedimentation effects which are not incorporated into either the elastic or plastic components of behaviour. For this reason, in the PM4Sand a postshaking function was implemented: this function reduces volumetric and shear moduli, increasing the reconsolidation strains to compensate the sedimentation strains. The parameters of PM4Sand were calibrated on the results of some laboratory test carried out on Ticino sand (Fioravante et al., 2016). The cyclic shear stress resistance curve simulation given by PM4Sand is reported in Figure 5: the numerical results are in a good accordance to the experimental data.

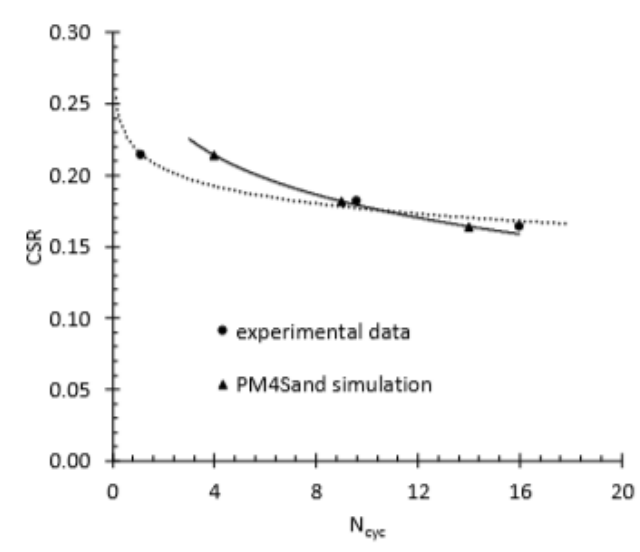

Figure 5. Comparison between cyclic resistance curves given by numerical simulation and by laboratory tests results (Ticino sand).

\section{UBCSAND}

UBC3D-PML (Petalas 2012) is a constitutive model, implemented in PLAXIS, based on the UBCSand (Puebla et al. 1997) that uses two yield surfaces of 
the Mohr-Coulomb type: the first surface is related to the isotropic hardening, while the second one is related to the kinematic hardening. UBCSand is an elastoplastic model with non associated plastic flow rule and it is based on the Drucker-Prager's low and Rowe's stress dilatancy hypothesis. Its formulation is based on classical plasticity theory with a hyperbolic strain hardening rule, based on the Duncan-Chang approach with modifications.

The hardening rule relates the mobilized friction angle to the plastic shear strain at a given stress. The flow rule in the model is based on the stress dilatancy theory developed by Rowe (1962), linearized and simplified according to energy. Moreover, a modified non-associated plastic potential function based on Drucker-Prager's criterion is used, in order to maintain the assumption of stress-strain coaxially in the deviatoric plane for a stress path beginning from the isotropic line. A soil densification rule is added in order to predict a more realistic evolution of excess pore pressures during cyclic loading. This allows the increase of the volumetric strains with a decreasing rate during shearing. The elastic behaviour which occurs within the yield surface is governed by a non-linear rule. Once the stress state reaches the yield surface, plastic behaviour is predicted as long as the stress point is not going immediately back into the elastic zone. The hardening rule governs the amount of plastic strain as a result of mobilization of the shear strength.

The model parameters calibration can be performed by using empirical relationships based on sand relative density (Beaty and Byrne 2011), which are based on the SPT number N1,60. The parameters of PM4Sand were calibrated on the results of some test carried out on Ticino sand (Fioravante et al., 2016). As showed in Figure 6, a good agreement with experimental data is achieved by UBCSand simulation.

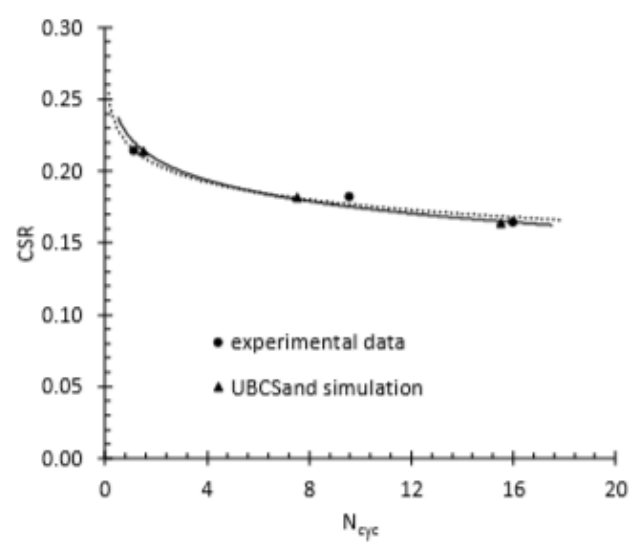

Figure 6. Comparison between cyclic resistance curves given by numerical simulation and by laboratory tests results (Ticino sand).

\subsection{Back analysis of centrifuge tests}

Some numerical analyses have been carried out with Plaxis2D software to verify the capability of advanced constitutive models (PM4sand and UBC3sand) to reproduce the phenomena observed in the centrifuge tests. The soil properties adopted in the numerical analyses are summarized in Tables 2 and 3.

Tied degrees of freedom between vertical sides were used as boundary conditions to reproduce the shear box used in the centrifuge. This option proposed by Zienkiewicz et al. (1988) connects the nodes on the same elevation at the left and right model boundaries. The nodes at the base of the finite element model were fixed in the vertical direction and a time history of acceleration was applied in the horizontal direction. Pore water pressure across the top surface is allowed whereas flow across the lateral boundaries is restricted.

The centrifuge tests performed in free field conditions have been simulated adopting the PM4sand constitutive model. The results of the simulations were shown (Fig.7) in terms of pore pressure ratio $\mathrm{Ru}$ obtained during the shaking (calculated at the shallowest point), and compared with the experimental data. It can be observed that the constitutive model is able to capture the liquefaction phenomenon ( $\mathrm{Ru}>0.8$ ) and the curves are in good agreement. The dissipation of pore pressure is major in the numerical model. 


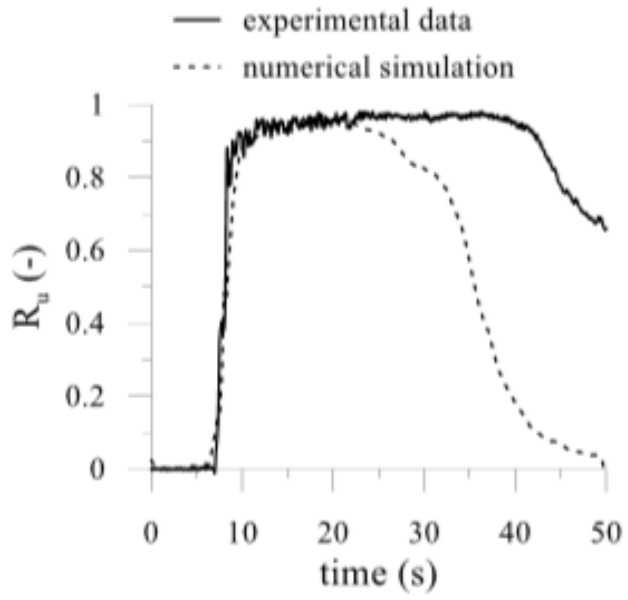

Figure 7. Pore pressure ratio Ru during the shaking: comparison between centrifuge tests data and numerical results (free field condition).

The centrifuge tests performed with the horizontal drains have been simulated adopting the UBCSand constitutive model. The numerical simulation results are reported in term of excess pore pressure ratio $(\mathrm{Ru})$ in Figure $8 \mathrm{a}$ for a $\mathrm{s} / \mathrm{d}=5$ (ratio between spacing and diameter of drains) and Figure $8 b$ for $s / d=10$.

It can be observed a very good agreement between experimental date and numerical simulation. A same peak of Ru is achieved in both cases, probably this event is due to the different depth of the pore pressure transducers, which in case $s / d=5$ is shallower that in case $s / d=10$.

The calibration of the numerical model allows to obtain a benchmark model which can be used for extrapolation of different geometrical layouts and parametrical study in order to obtain indications for the design of the most effective mitigation techniques.
Table 2. PM4sand parameters

\begin{tabular}{llll}
\hline Parameter & Description & Value & Unit \\
\hline $\mathrm{G}_{0}$ & Shear modulus coefficient & 624 & - \\
\hline $\mathrm{h}_{0}$ & Contraction rate parameter & 0.1 & - \\
\hline $\mathrm{p}_{\mathrm{A}}$ & Atmospheric pressure & 101.3 & $\mathrm{kN} / \mathrm{m}^{2}$ \\
\hline $\mathrm{e}_{\max }$ & maximum void ratio & 0.923 & - \\
\hline $\mathrm{e}_{\min }$ & minimum void ratio & 0.574 & - \\
\hline $\mathrm{n}_{\mathrm{b}}$ & Bounding surface parameter & 0.5 & - \\
\hline $\mathrm{n}_{\mathrm{d}}$ & Dilatancy surface parameter & 0.1 & - \\
\hline$\phi_{\mathrm{cv}}$ & critical state friction angle & 33 & - \\
\hline $\mathrm{nu}$ & Poisson's ratio & 0.3 & - \\
\hline $\mathrm{Q}$ & critical state line parameter & 8 & - \\
\hline $\mathrm{R}$ & critical state line parameter & 0.6 & - \\
\hline
\end{tabular}

Table 3. UBCsand parameters

\begin{tabular}{clll}
\hline Parameter & Description & Value & Unit \\
\hline$N_{1,60}$ & Correct blow count & 624 & - \\
\hline$v$ & Poisson's ratio & 0.3 & - \\
\hline$K_{B}^{e}$ & $\begin{array}{l}\text { Elastic bulkmodulus } \\
\text { factor }\end{array}$ & 2246.0 & - \\
\hline$K_{G}^{e}$ & $\begin{array}{l}\text { Elastic shear modulus } \\
\text { factor }\end{array}$ & 1036.0 & - \\
\hline$K_{G}^{p}$ & $\begin{array}{l}\text { Plastic shear modulus } \\
\text { factor }\end{array}$ & 680.6 & - \\
\hline$m_{e}$ & Elasticity factor & 0.5 & - \\
\hline$n_{e}$ & Elasticity factor & 0.5 & - \\
\hline$n_{p}$ & Plasticity factor & 0.4 & - \\
\hline$\phi_{C v}$ & Poisson's ratio & 33.0 & - \\
\hline$\phi_{\text {peak }}$ & $\begin{array}{l}\text { critical state line } \\
\text { parameter }\end{array}$ & 34.4 & - \\
\hline$R_{f}$ & $\begin{array}{l}\text { critical state line } \\
\text { parameter }\end{array}$ & 0.74 & - \\
\hline$f_{\text {dens }}$ & Densification factor & 9.0 & - \\
\hline$f_{E, p o s t}$ & Post-liquefaction factor & 1.0 & - \\
\hline & &
\end{tabular}




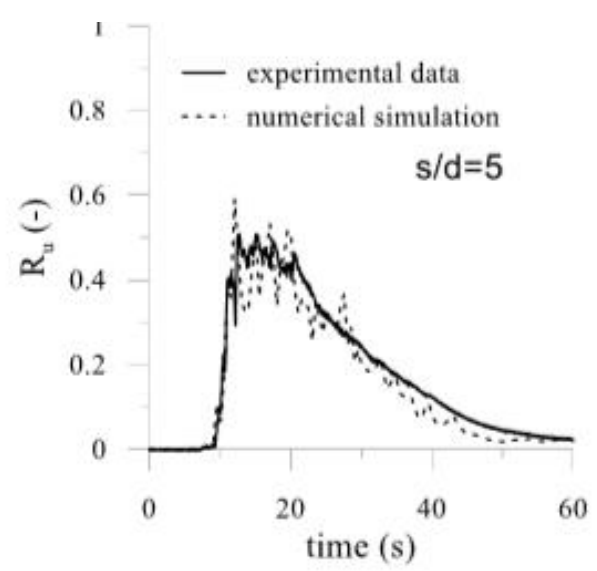

(a)

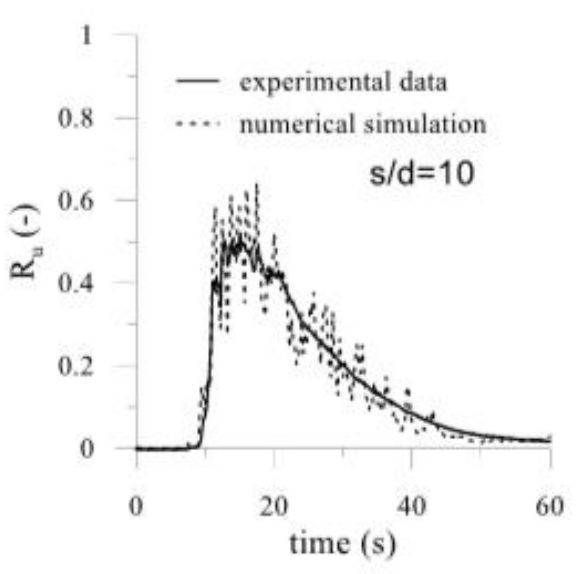

(b)

Figure 8. Excess pore pressure ratio (ru) time history for disposition of horizontal drains with $s / d=5$ (a) and $s / d=10(b)$.

\section{Concluding remarks}

A large experimental programme has been developed at different scales (laboratory tests, centrifuge tests, in situ tests) in order to analyse and to compare the effectiveness of some liquefaction mitigation techniques. The experimental results allowed to calibrate advanced numerical models and to build up a benchmark model which will be used for extrapolation of different geometrical layouts in order to obtain indications for the design of such techniques.

\section{ACKNOWLEDGEMENTS}

This work was carried out as part of the European project Horizon 2020 - Assessment and Mitigation of liquefaction potential across Europe: A holistic approach to protect structures infrastructures for improved resilience to earthquake - induced liquefaction disasters - "LIQUEFACT" (grant agreement No. 700748).

\section{References}

[1] Dafalias, Y. F., \& Manzari, M. T. Simple plasticity sand model accounting for fabric change effects. Journal of Engineering mechanics 2004, 130(6), 622-634.

[2] El Mohtar C.S., Bobet A., Drnevich V.P., Johnston C.T., Santagata M.C. Pore pressure generation in sand with bentonite: from small strains to liquefaction. Gèotechnique, 2014, 64 (2): 108-107.

[3] Flora A., Bilotta E., Chiaradonna A., Fasano G., Lirer S., Mele L., Pingue L. Ground improvement to mitigate liquefaction potential: experimental evidences and design procedures. Proc. of the 7th Int. Conf. on Earth. Geotech. Eng.

[4] Fioravante V. \&Giretti D. Unidirectional cyclic resistance of Ticino and Toyoura sands from centrifuge cone penetration tests. Acta Geotechnica 2016, 11(4): 953- 968.

[5] Fioravante V., E. Bilotta, A.Chiaradonna, G. Fasano, A. Flora, Lirer L., L. Mele, V. Nappa (2019). Centrifuge modelling of soilstructure interaction in liquefiable ground before and after the application of remediation tecniques. Proc. of VII International Conference on Earthquake Geotechnical Engineering ICEGE 2019, 17-20 June Roma (Italy).

[6] Huang Y. e Wang L. Laboratory investigation of liquefaction mitigation in silty sand using laponite. Engineering Geology, 2016, 204, 23-32.

[7] Idriss, I. M., \& Boulanger, R. W. Soil liquefaction during earthquakes. Earthquake Engineering Research Institute 2008.

[8] Ishihara K. and Koseki J. Discussion of cyclic shear strength of fines-containing sands. Proc., 12th Int. Conf. on Soil Mech. Geotech. Eng. 1989, London, 101-106.

[9] Ishihara K. Liquefaction and flow failure during earthquakes. Gèotechnique, 1993,43 (3): 351-415. 
[10] Ishihara K., Tsukamoto Y., Nakazawa H., Kamada K., and Huang Y. Resistance of partly saturated sand to liquefaction with reference to longitudinal and shear wave velocities. Soil Foundation., 2002, 42(6): 93105.

[11] Manzari, M. T., \& Dafalias, Y. F. A critical state two-surface plasticity model for sands. Geotechnique 1997, 47(2), 255-272.

[12] Mele L., Flora A., Lirer S., d'Onofrio A., Bilotta E. (2018). Experimental study of the injectability and effectiveness of laponite mixtures as liquefaction mitigation technique. Proc. 5th Geotechnical Earthquake Engineering and soil dynamics, GEESDV, Austin, Texas (US), June 2018.

[13] Ochoa-Cornejo F., Bobet A., Johnston C. T., Santagata M., Sinfield J. V. Cyclic behaviour and pore pressure generation in sands with laponite, a super-plastic nanoparticle. Soil Dynamics and Earthquake Engineering, 2016, 88, 265-279.

[14] Okamura M. and Soga Y. Effects of pore fluid compressibility on liquefaction resistance of partially saturated sand. Soils and Foundations, 2006, 46: 695-700.

[15] Petalas, A., \&Galavi, V. Plaxis Liquefaction Model UBC3DPLM. PLAXIS Report 2013.

[16] Puebla, H., Byrne, P. M., \& Phillips, R. Analysis of CANLEX liquefaction embankments: prototype and centrifuge models. Canadian Geotechnical Journal 1997, 34(5), 641-657.

[17] Yegian M. K., Eseller-Bayat E., Alshawabkeh A. and Ali S. Induced-Partial Saturation for liquefaction mitigation: experimental investigation. Journal of geotechnical and geoenviromental engineering ASCE, 2007,133(4): 372-380.

[18] Zienkiewicz, O.C., Bicanic, N., Shen, F.Q. (1988). Earthquake input definition and the transmitting boundary condition. Advances in Computational Nonlinear Mechanics I, 109-138 